\title{
MANIFOLDS WITH LARGE ISOTROPY GROUPS
}

\author{
ANDREAS KOLLROSS AND EVANGELIA SAMIOU
}

\begin{abstract}
We classify all simply connected Riemannian manifolds whose isotropy groups act with cohomogeneity less than or equal to two.
\end{abstract}

\section{INTRODUCTION AND RESUlts}

Let $M$ be a simply connected Riemannian manifold and $G$ a closed connected subgroup of the isometry group of $M$. We consider the action of an isotropy group, i.e. the subgroup $G_{p}=\{g \in G \mid g \cdot p=p\}$ consisting of all elements which leave one point $p \in M$ fixed. This group $G_{p}$ acts on $M$ by restriction of the $G$-action and by the differentials of the isometries in $G$ on the tangent space $\mathrm{T}_{p} M$; we call the first the isotropy action, while the latter is called isotropy representation. For any differentiable Lie group action, the minimal orbit codimension is called the cohomogeneity of the action.

It is a well known classical result that two-point homogeneous spaces, i.e. Riemannian manifolds where any ordered pair of equidistant points can be mapped to any other such pair by an isometry, are exactly the rank-one symmetric and Euclidean spaces [5]. Twopoint homogeneity is equivalent to the condition that the isotropy action at any point is of cohomogeneity one.

Thus, Riemannian manifolds whose isotropy actions are of cohomogeneity one are wellknown and it is a natural question to ask which Riemannian manifolds have isotropy actions of low cohomogeneity. In this article, we classify all simply connected Riemannian manifolds $M$ whose isotropy groups act with cohomogeneity less than or equal to two. For homogeneous spaces this condition is equivalent to saying that the unit tangent bundle of $M$ is of cohomogeneity one. However, there are also non-homogeneous examples of such manifolds.

By $K=G_{p}$ we will usually denote the isotropy subgroup of the $G$-action at a point $p$ lying in a principal orbit. Furthermore we denote by $c(G, M)$ the cohomogeneity of the $G$-action on $M$. For the cohomogeneity of the $G$-action on the tangent bundle we have

$$
c(G, T M)=c\left(K, T_{p} G p\right)+2 c(G, M)=c(K, M)+c(G, M) .
$$

Date: October 29, 2018.

2000 Mathematics Subject Classification. 53C30, 22E46, 57S15.

Key words and phrases. homogeneous spaces, isotropy representation, low cohomogeneity.

Research supported by the University of Cyprus. 
Our main result for the homogeneous case is given by the following theorem. For the non-homogeneous case, see Theorem 5.1. Of course, if we allow the isotropy group to be trivial, we get all two-dimensional manifolds.

Theorem 1.1. Let $M=G / K$ be a simply connected Riemannian homogeneous space such that the isotropy group $K$ acts with cohomogeneity two on $M$. Assume further that $M$ is de Rham irreducible. Then $M$ is isometric to one of the following homogeneous spaces equipped with a G-invariant metric.

(i) An irreducible symmetric space of rank two.

(ii) A generalized Heisenberg group of type

$$
N(1, n), N(2, n), N(3 ; n, 0), N(6,1), N(7 ; 1,0) .
$$

(iii) One of the following compact homogeneous spaces:

$$
\frac{\mathrm{SU}(n+1)}{\mathrm{SU}(n)}, \frac{\mathrm{Sp}(n+1)}{\mathrm{U}(1) \cdot \operatorname{Sp}(n)}, \frac{\mathrm{Sp}(1) \cdot \mathrm{Sp}(n+1)}{\Delta \mathrm{Sp}(1) \cdot \operatorname{Sp}(n)}, \frac{\operatorname{Spin}(9)}{\operatorname{Spin}(7)} .
$$

(iv) One of the following non-compact homogenous spaces:

$$
\begin{gathered}
\frac{\mathrm{SU}(n, 1)}{\mathrm{SU}(n)}, \frac{\operatorname{Sp}(n, 1)}{\mathrm{U}(1) \cdot \operatorname{Sp}(n)}, \frac{\operatorname{Sp}(1) \cdot \operatorname{Sp}(n) \ltimes \mathbb{R}^{4 n}}{\mathrm{U}(1) \cdot \operatorname{Sp}(n)}, \frac{\operatorname{Sp}(1) \cdot \operatorname{Sp}(n, 1)}{\Delta \operatorname{Sp}(1) \cdot \operatorname{Sp}(n)}, \\
\frac{\operatorname{Sp}(1) \cdot\left(\operatorname{Sp}(1) \cdot \operatorname{Sp}(n) \ltimes \mathbb{R}^{4 n}\right)}{\Delta \operatorname{Sp}(1) \cdot \operatorname{Sp}(n)}, \frac{\operatorname{Spin}(7) \ltimes \delta_{7}}{\operatorname{Spin}(6)}, \frac{\operatorname{Spin}(8,1)}{\operatorname{Spin}(7)}, \frac{\operatorname{Spin}(8) \ltimes \delta_{8}^{+}}{\operatorname{Spin}(7)} .
\end{gathered}
$$

(v) Hyperbolic space with G-action as given in Theorem 3.4 .

The paper is organized as follows. We start with a splitting criterion for homogeneous spaces which we use to detect Riemannian products from the isotropy representation. In Section 3 we prove fibration theorems for homogeneous spaces with reducible isotropy representation with two submodules. The actual classification of homogeneous spaces with isotropy cohomogeneity two is done in Section 4. We first determine all effective representations of cohomogeneity two. Using the splitting criterion from Section 2, we eliminate those leading to Riemannian products. Then we construct all homogeneous spaces corresponding to the remaining representations. The final Section 5 contains the classification in the inhomogeneous case.

We would like to thank Carlos Olmos for helpful discussions.

\section{A SPlitting CRITERION FOR HOMOgEneOUs SPACES}

If $M$ is isometric to a product $M=X \times Y$, with $X$ irreducible or maximal Euclidean then the factors $X$ and $Y$ are preserved by any connected group of isometries. In particular, the action of any connected group of isometries on $M$ projects to actions of lower cohomogeneity on both factors $X$ and $Y$. Furthermore, if $M$ is homogeneous then so are $X$ and $Y$. For the classification we may therefore exclude reducible Riemannian manifolds. These can be detected by means of Corollary 2.4 from the isotropy representation. 
Lemma 2.1. Let $M=G / K$ be a Riemannian homogeneous space and $V \subseteq T_{p} M$ a submodule of the isotropy representation. Assume that $V$ is the fixed point set of some subgroup $N \subseteq K$. Then the $G$-invariant distribution $\mathcal{D}_{V}$ given by

$$
\mathcal{D}_{V}(g p)=d_{p} g V
$$

is well-defined and integrable. The leaf through $p \in M$ is a connected component of the fixed point set of $N$ and therefore the leaves of the foliation corresponding to $\mathcal{D}_{V}$ are closed totally geodesic submanifolds.

Proof. It follows from the $K$-invariance of $V$ that the distribution $\mathcal{D}$ is well-defined. We may assume that $N \subseteq K$ is the kernel of the $K$-action $K \rightarrow \mathrm{SO}(V)$ on $V$ and let $F \subseteq M$ be the connected component containing $p$ of the fixed point set of the $N$-action on $M$. Thus $F \subset M$ is a totally geodesic submanifold and $T_{p} F=V$.

We are going to show that $F$ is everywhere tangent to the distribution $\mathcal{D}$. For $g p=q \in F$, let

$$
V_{g}=g^{-1} T_{q} F \subseteq T_{p} M \quad \text { and } \quad N_{g}=g^{-1} N g \subseteq K .
$$

Clearly, $N_{g}$ fixes $V_{g}$ pointwisely, i.e. $n u=u$ for each $n \in N_{g}$ and $u \in V_{g}$. Also, since $N_{g} \subseteq K$, it preserves the decomposition $T_{p} M=V \oplus V^{\perp}$ of $T_{p} M$ in $K$-invariant submodules, i.e. $N_{g} V=V$ and $N_{g} V^{\perp}=V^{\perp}$. We have constructed a continuous map

$$
g \mapsto V_{g} \in G_{\operatorname{dim}(V)}\left(V \oplus V^{\perp}\right)
$$

into the Grassmannian sending the identity element $e \in G$ to $V_{e}=V$. A chart around $V$ for this Grassmannian is given by

$$
\operatorname{Hom}\left(V, V^{\perp}\right) \rightarrow G_{\operatorname{dim}(V)}\left(V \oplus V^{\perp}\right), \quad A \mapsto \Gamma(A)=\{(v, A v) \mid v \in V\} .
$$

Hence for $g$ sufficiently close to $e$ we can write

$$
V_{g}=\left\{\left(v, A_{g} v\right) \mid v \in V\right\}
$$

with $A_{g} \in \operatorname{Hom}\left(V, V^{\perp}\right)$ depending continuously on $g$ and $A_{e}=0$. Since $N_{g}$ fixes $V_{g}$ pointwisely we have for $n \in N_{g}$ and $v \in V$ that

$$
\left(n v, n A_{g} v\right)=n\left(v, A_{g} v\right)=\left(v, A_{g} v\right)
$$

and thus $n v=v$, and $n A_{g} v=A_{g} v$. Therefore $N_{g} \subseteq N$ and, since $N_{g}, N$ are isomorphic compact Lie groups, we actually have $N_{g}=N$. From (2.3) we now have $n A_{g} v=A_{g} v$ for all $n \in N$. Thus $A_{g} v \in V \cap V^{\perp}=0$.

We have shown that $V_{g}=V$ as long as $V_{g}$ is in the image $U_{0}$ of the map (2.2). Since $F$ is connected, for each $q \in F$ there is a path $g(t) \in G, t \in[0,1]$ such that $g(t) p \in F, g(1) p=q$ and $g(0)=e$. The set $\left\{t \in[0,1] \mid V_{g(t)} \in U_{0}\right\} \subset[0,1]$ is open and closed since, by the above, " $V_{g(t)} \in U_{0}$ " is equivalent to " $V_{g(t)}=V$ ". Hence $V_{g}=V$ for all $g \in G$ with $g p \in F$. In particular, $T_{q} F=g V=\mathcal{D}_{V}(q)$.

Lemma 2.1 provides a splitting criterion for simply connected homogeneous spaces with reducible isotropy representation. 
Corollary 2.4. Let $M=G / K$ be an effectively presented simply connected Riemannian homogeneous space. Assume that the isotropy representation

$$
T_{p} M=\mathfrak{m}=\mathfrak{m}_{1} \oplus \mathfrak{m}_{2}
$$

splits with nontrivial $K$-submodules $\mathfrak{m}_{1}, \mathfrak{m}_{2}$. Additionally suppose that both $\mathfrak{m}_{1}$, $\mathfrak{m}_{2}$ are fixed modules of suitable subgroups of $K$, i.e. there are subgroups $N_{i} \subset K$ such that

$$
\mathfrak{m}_{i}=\operatorname{Fix}\left(N_{i}\right)=\left\{v \in \mathfrak{m} \mid n v=v \text { for all } n \in N_{i}\right\} .
$$

Then $M$ admits a nontrivial de Rham decomposition $M=M_{1} \times M_{2}$.

Proof. We have subgroups $N_{i}=\operatorname{ker}\left(K \rightarrow \mathrm{SO}\left(\mathfrak{m}_{i}\right)\right) \subset K$ such that

$$
\mathfrak{m}_{i}=\left\{x \in \mathfrak{m} \mid n x=x \text { for all } n \in N_{i}\right\} .
$$

By Lemma 2.1 we have complementary integrable totally geodesic $G$-invariant distributions $\mathcal{D}_{\mathfrak{m}_{i}}, i=1,2$,

$$
T M=\mathcal{D}_{\mathfrak{m}_{1}} \oplus \mathcal{D}_{\mathfrak{m}_{2}}
$$

By $G$-invariance, these distributions are perpendicular at each point $q \in M$. The distributions are also parallel. To see this, let $x, y \in \Gamma \mathcal{D}_{\mathfrak{m}_{1}}$ and $v, w \in \Gamma \mathcal{D}_{\mathfrak{m}_{2}}$ be sections and compute

$$
\begin{aligned}
& \left\langle\nabla_{x} v \mid y\right\rangle=-\left\langle v \mid \nabla_{x} y\right\rangle=-\left\langle v \mid \mathrm{II}_{1}(x, y)\right\rangle=0 \quad \text { and } \\
& \left\langle\nabla_{w} v \mid y\right\rangle=\left\langle\mathrm{II}_{2}(w, v) \mid y\right\rangle=0
\end{aligned}
$$

where $\mathrm{II}_{i}$ denotes the second fundamental form of $\mathcal{D}_{\mathfrak{m}_{i}}$. This shows that $\nabla_{x+w} v \in \mathcal{D}_{\mathfrak{m}_{i}}$ for each $x+w \in T M$ and each section $v \in \Gamma \mathcal{D}_{\mathfrak{m}_{i}}$. Thus (2.5) is preserved by the holonomy of $M$ at $p$. By the de Rham Holonomy Theorem $M=M_{1} \times M_{2}$ splits as a Riemannian product according to (2.6).

\section{Splitting isotropy And structure of homogeneous spaCes}

Theorem 3.1. Let $M=G / K$ be a Riemannian homogeneous space whose isotropy representation can be decomposed into two (not necessarily irreducible) invariant subspaces

$$
T_{p} M=\mathfrak{m}=\mathfrak{m}_{1} \oplus \mathfrak{m}_{2}
$$

such that the kernel $N_{1}$ of the $K$-representation on $\mathfrak{m}_{1}$ acts without nonzero fixed vectors on $\mathfrak{m}_{2}$. Then $\mathfrak{g}_{1}=\mathfrak{k} \oplus \mathfrak{m}_{1}$ is a subalgebra of the Lie algebra $\mathfrak{g}$ and the corresponding connected subgroup $G_{1}$ of $G$ is closed.

Proof. We first show that $\mathfrak{g}_{1}=\mathfrak{k} \oplus \mathfrak{m}_{1}$ is a Lie algebra. Clearly $[\mathfrak{k}, \mathfrak{k}] \subseteq \mathfrak{k} \subseteq \mathfrak{g}_{1}$ and $\left[\mathfrak{k}, \mathfrak{m}_{1}\right] \subseteq$ $\mathfrak{m}_{1} \subseteq \mathfrak{g}_{1}$. It remains to show that $\left[\mathfrak{m}_{1}, \mathfrak{m}_{1}\right] \subseteq \mathfrak{g}_{1}$, or, equivalently, that for $m, m^{\prime} \in \mathfrak{m}_{1}$ we have $\left[m, m^{\prime}\right] \perp \mathfrak{m}_{2}$. To this end, let pr: $\mathfrak{g} \rightarrow \mathfrak{m}_{2}$ be the orthogonal projection. Since $N_{1} \subseteq K$ and $K$ acts isometrically on $\mathfrak{g}$, this map is $N_{1}$-equivariant. Now for $n \in N_{1}$ we compute

$$
\operatorname{Ad}_{n} \operatorname{pr}\left[m, m^{\prime}\right]=\operatorname{pr} \operatorname{Ad}_{n}\left[m, m^{\prime}\right]=\operatorname{pr}\left[\operatorname{Ad}_{n} m, \operatorname{Ad}_{n} m^{\prime}\right]=\operatorname{pr}\left[m, m^{\prime}\right] .
$$

Hence $\operatorname{pr}\left[m, m^{\prime}\right]$ is a fixed vector for $N_{1}$ in $\mathfrak{m}_{2}$ and therefore zero. 
In order to show that the connected subgroup $G_{1} \subseteq G$ with Lie algebra $\mathfrak{g}_{1}$ is closed, recall that in the isotropy representation, $\mathfrak{m}_{1}$ is the fixed point set of $N_{1}$. Thus by Lemma 2.1 the distribution $\mathcal{D}_{\mathfrak{m}_{1}}$ is integrable with closed totally geodesic leaves. The orbit $G_{1} p$ is an immersed submanifold of $M$. We also have that $\mathcal{D}_{\mathfrak{m}_{1}}(p)=\mathfrak{m}_{1}=T_{p} G_{1} p$ and, by left invariance of the distribution $\mathcal{D}_{\mathfrak{m}_{1}}$, that $\mathcal{D}_{\mathfrak{m}_{1}}(q)=T_{q} G_{1} p$ for each $q=g_{1} p \in G_{1} p$. Since $G_{1} p$ is connected, it is contained in the leaf $F$ of $\mathcal{D}_{\mathfrak{m}_{1}}$ through $p$. We now show that $G_{1} p=F$. Since both have the same dimension $\operatorname{dim} \mathfrak{m}_{1}$, there is $r>0$ such that $F \cap B_{r}(p) \subseteq G_{1} p$, where $B_{r}(p)=\{x \in M \mid d(x, p)<r\}$. If $q=g_{1} p \in G_{1} p$, then $g_{1} F=F$ and $F \cap B_{r}(q)=$ $g_{1}\left(F \cap B_{r}(p)\right) \subseteq g_{1} G_{1} p=G_{1} p$. Thus $G_{1} p$ has the following property:

$$
q \in G_{1} p \Rightarrow F \cap B_{r}(q) \subseteq G_{1} p
$$

But then also the complement $F \backslash G_{1} p$ has this property. It follows that both $G_{1} p$ and $F \backslash G_{1} p$ are open in $F$. Since $F$ is connected, we have $G_{1} p=F$. Now $G_{1}$ is closed, since it is the preimage $G_{1}=\operatorname{pr}^{-1}\left(G_{1} p\right)$ under the projection pr: $G \rightarrow G / K=M, \operatorname{pr}: g \mapsto g p=$ $g K \in M$.

In general, the adjoint representation of $G$ restricted to $G_{1}$ does not preserve $\mathfrak{m}_{2}$, but induces an action on $\mathfrak{g} / \mathfrak{g}_{1}$ by

$$
G_{1} \times \mathfrak{g} / \mathfrak{g}_{1} \ni\left(x, m+\mathfrak{g}_{1}\right) \mapsto \operatorname{Ad}_{x} m+\mathfrak{g}_{1} \in \mathfrak{g} / \mathfrak{g}_{1} .
$$

Under the isomorphism

$$
\begin{aligned}
\mathfrak{m}_{2} & \cong \mathfrak{g} / \mathfrak{g}_{1} \\
m & \mapsto m+\mathfrak{g}_{1}
\end{aligned}
$$

and the orthogonal projection pr: $\mathfrak{g} \rightarrow \mathfrak{m}_{2}$ this induces a $G_{1}$-action on $\mathfrak{m}_{2}$ given by

$$
G_{1} \times \mathfrak{m}_{2} \ni(x, m) \mapsto \operatorname{pr}\left(\operatorname{Ad}_{x} m\right)
$$

which extends the action $K \rightarrow \mathrm{SO}\left(\mathfrak{m}_{2}\right)$ of $K$. Henceforth we will refer to this action simply as the $G_{1}$-action on $\mathfrak{m}_{2}$.

Theorem 3.3. Let $M=G / K$ be as in Theorem 3.1 and assume the $G_{1}$-action on $\mathfrak{m}_{2}$ is isometric. Then the quotient $B=G / G_{1}$ is a Riemannian homogeneous space and we have a Riemannian submersion and homogeneous fibre bundle

$$
F=G_{1} / K \hookrightarrow M=G / K \stackrel{\pi}{\longrightarrow} G / G_{1}=B
$$

with structure group $G_{1}$, base $B$ and totally geodesic fibres $F$.

Proof. Assume that $G_{1}$ acts isometrically on $\mathfrak{m}_{2}$. The scalar product on $\mathfrak{m}_{2} \cong \mathfrak{g} / \mathfrak{g}_{1} \cong T_{G_{1}} B$ then extends to a left invariant metric on $B=G / G_{1}$ and the projection $G / K \rightarrow G / G_{1}$ becomes a Riemannian submersion.

Let us now look at the case where $G_{1}$ does not act isometrically on $\mathfrak{m}_{2}$. 
Theorem 3.4. Let $M=G / K$ be as in Theorem 3.1 and such that the $G_{1}$-action on $\mathfrak{m}_{2}$ is not isometric. Assume that $K$ is irreducible on $\mathfrak{m}_{2}$ and for all $m \in \mathfrak{m}_{2}$ the isotropy subgroup $K_{m}$ of the $K$-action on $\mathfrak{m}_{2}$ acts irreducibly on $\mathfrak{m}_{1}$. Then $\mathfrak{m}_{1}$ is one-dimensional and $M$ is a semidirect product

$$
M=\mathbb{R} \ltimes \mathbb{K}^{n} \quad \text { where } \mathbb{K}=\mathbb{R}, \mathbb{C} \text {, or } \mathbb{H}
$$

endowed with a left invariant metric arising from the orthogonal direct sum of standard scalar products on $\mathbb{R}$ and $\mathbb{K}^{n}$. In (3.5) the $\mathbb{R}$-factor acts on $\mathbb{K}^{n}$ via a group monomorphism $\mathbb{R} \rightarrow \mathbb{K}^{\times}, t \mapsto e^{\lambda t}$, for some not purely imaginary $\lambda \in \mathbb{K}$ and $\mathbb{K}^{n}$ carries the standard scalar product.

In particular, up to rescaling, $M$ is isometric to hyperbolic space.

Proof. Suppose $G_{1}$ does not act isometrically on $\mathfrak{m}_{2}$. Since $G_{1}$ is connected, there is $\xi \in \mathfrak{g}_{1}$ such that the endomorphism of $\mathfrak{m}_{2}$ mapping $m \mapsto \operatorname{pr}[\xi, m]$ is not skew-symmetric, i.e. such that $\langle m \mid[\xi, m]\rangle \neq 0$ for some, and therefore almost all, $m \in \mathfrak{m}_{2}$ and $\xi \in \mathfrak{m}_{1}$. Since $\mathfrak{g}_{1}=\mathfrak{k} \oplus \mathfrak{m}_{1}$ and $\operatorname{ad}_{\mathfrak{k}}$ is skew-symmetric on $\mathfrak{m}_{2}$ we may assume $\xi \in \mathfrak{m}_{1}$. We fix such an $m \in \mathfrak{m}_{2},|m|=1$ and consider the homomorphism

$$
\mathfrak{m}_{1} \rightarrow \mathbb{R}, \quad \xi \mapsto\langle m \mid[\xi, m]\rangle
$$

which is not zero. By the assumption, the isotropy group $K_{m}$ acts irreducibly on $\mathfrak{m}_{1}$, and, since (3.6) is $K_{m}$-equivariant, Schur's Lemma yields an isomorphism $\mathfrak{m}_{1} \cong \mathbb{R}$.

We have $G_{1}=K \cdot Q$ with a one-dimensional group $Q \cong \mathrm{S}^{1}$ or $\cong \mathbb{R}_{+}$. For $x \in Q$ consider the adjoint $\operatorname{Ad}_{x}$ of $x$ acting on $\mathfrak{g}=\mathfrak{g}_{1} \oplus \mathfrak{m}_{2} \ni y+m$. We have

$$
\operatorname{Ad}_{x}(y+m)=y+A_{x} m+L_{x} m
$$

with homomorphisms $A_{x} \in \operatorname{Hom}\left(\mathfrak{m}_{2}, \mathfrak{g}_{1}\right)$ and $L_{x} \in \operatorname{Hom}\left(\mathfrak{m}_{2}, \mathfrak{m}_{2}\right)$. Since $\operatorname{Ad}_{x}$ commutes with all $\operatorname{Ad}_{k}, k \in K$, the homomorphisms $A_{x}$ and $L_{x}$ are $K$-module homomorphisms. It follows from Schur's Lemma that

$$
L_{x} \in \operatorname{End}\left(\mathfrak{m}_{2}\right)^{K} \cong \mathbb{K}=\mathbb{R}, \mathbb{C}, \mathbb{H}
$$

and that $A_{x}: \mathfrak{m}_{2} \rightarrow \mathfrak{g}_{1}=\mathfrak{k} \oplus \mathbb{R}$ is zero, an isomorphism $\mathfrak{m}_{2} \cong \mathbb{R}$ or an isomorphism of $\mathfrak{m}_{2}$ with some nontrivial irreducible submodule in the adjoint representation of $K$. If $\mathfrak{m}_{2} \cong \mathbb{R}$, then $K$ is trivial and $M=G$ is a two-dimensional Lie group. In this case it follows that $M$ is isomorphic to $\mathbb{R}_{+} \ltimes \mathbb{R}$, where the left factor acts by multiplication on the right, since there is only one isomorphism class of non-abelian two-dimensional real Lie algebras.

Thus we may assume $A_{x}: \mathfrak{m}_{2} \rightarrow \mathfrak{k}$. We have a one-parameter group

$$
L: Q \rightarrow \mathbb{K}^{\times}, \quad x \mapsto L_{x}
$$

into the multiplicative group of the (skew) field $\mathbb{K}$. Let $\xi \in \mathfrak{m}_{1} \cong \mathbb{R}$ be an infinitesimal generator of $Q$. Differentiating (3.7) we have

$$
\operatorname{ad}_{\xi}(y+m)=d A(\xi) m+d L(\xi) m
$$


with $d L$ nontrivial, since $G_{1}$ and therefore $Q$ is not isometric on $\mathfrak{m}_{2}$. Thus $0 \neq \lambda=d L(\xi) \in \mathbb{K}$ and we can diagonalize $\operatorname{ad}_{\xi}$ on $\mathfrak{g}$. In fact, the eigenspaces are

$$
\begin{aligned}
& E\left(\operatorname{ad}_{\xi}, 0\right)=\mathfrak{g}_{1}=\mathfrak{k} \oplus \mathbb{R}, \\
& E\left(\operatorname{ad}_{\xi}, \lambda\right)=\left\{\lambda^{-1} d A(\xi) m+m \mid m \in \mathfrak{m}_{2}\right\} \subseteq \mathfrak{k} \oplus \mathfrak{m}_{2} .
\end{aligned}
$$

Since $Q$ commutes with $K$ we have $\operatorname{Ad}_{K} \xi=\xi$ and therefore the eigenspaces (3.9) are $\operatorname{Ad}_{K^{-}}$ invariant. In particular, we may replace $\mathfrak{m}_{1} \oplus \mathfrak{m}_{2}$ by $\mathfrak{m}_{1} \oplus E\left(\operatorname{ad}_{\xi}, \lambda\right)$ as an $\operatorname{Ad}_{K}$-invariant complement of $\mathfrak{k}$ in $\mathfrak{g}$ to describe the metric structure of $G / K$. In other words, we may assume w.l.o.g. $\mathfrak{m}_{2}=E\left(\operatorname{ad}_{\xi}, \lambda\right), d A(\xi)=0$ and thus $A_{x}=0$ for all $x \in Q$.

As $G_{1}$ is not isometric on $\mathfrak{m}_{2}$ the image of the 1-parameter group $L$ is not contained in the unit sphere of $\mathbb{K}$. Hence $Q=\mathbb{R}$, and we find $x \in Q$ such that $L_{x}=\lambda \in \mathbb{R} \subset \mathbb{K},|\lambda| \neq 1$. For this $x$ we consider the automorphism $\operatorname{Ad}_{x}$ of the Lie algebra $\mathfrak{g}$. For $m_{1}, m_{2} \in \mathfrak{m}_{2}$ we compute

$$
\begin{aligned}
\operatorname{Ad}_{x}\left(\left[m_{1}, m_{2}\right]\right) & =\left[m_{1}, m_{2}\right]_{\mathfrak{g}_{1}}+\lambda\left[m_{1}, m_{2}\right]_{\mathfrak{m}_{2}} \\
=\left[\operatorname{Ad}_{x} m_{1}, \operatorname{Ad}_{x} m_{2}\right] & =\lambda^{2}\left[m_{1}, m_{2}\right]_{\mathfrak{g}_{1}}+\lambda^{2}\left[m_{1}, m_{2}\right]_{\mathfrak{m}_{2}}
\end{aligned}
$$

Since $|\lambda| \neq 1$ we conclude that $\left[m_{1}, m_{2}\right]=0$ and $\mathfrak{m}_{2}$ is an abelian ideal in $\mathfrak{g}$. It follows that $\mathfrak{m}_{1} \oplus \mathfrak{m}_{2}$ is a solvable ideal in $\mathfrak{g}$.

The connected abelian subgroup $M_{2} \subset G$ corresponding to $\mathfrak{m}_{2}$ cannot have compact factors since its automorphism group contains $K$, which acts irreducibly on the Lie algebra $\mathfrak{m}_{2}$. Hence $M_{2}=\mathfrak{m}_{2} \cong \mathbb{K}^{\ell}$ is a $\mathbb{K}$-vector space and the subgroup of $G$ corresponding to $\mathfrak{m}_{1} \oplus \mathfrak{m}_{2}$ is the semidirect product $Q \ltimes_{L} M_{2}$, which acts transitively on the simply connected manifold $M$ with discrete isotropy groups. It follows that $Q \ltimes_{L} M_{2}$ acts simply transitively on $M$ and we may identify $M$ with the group $Q \ltimes_{L} M_{2}$. Hence we have $M=Q \ltimes_{L} M_{2}=\mathbb{R} \ltimes_{L} \mathbb{K}^{\ell}$, where $L$ is the group homomorphism (3.8).

To prove that $M$ is isometric to hyperbolic space we only need that $L(t)=e^{\lambda t} \omega(t)$ with $\lambda \in \mathbb{R}$ and a one-parameter group $\omega(t) \in \mathrm{O}\left(\mathfrak{m}_{2}\right)$ of isometries of $\mathfrak{m}_{2}$. Identifying $M$ with the product $\mathbb{R} \times \mathfrak{m}_{2}$, the group structure is given by

$$
(t, x) \cdot(s, y)=\left(t+s, x+e^{\lambda t} \omega(t) y\right)
$$

and the left invariant metric is

$$
g(t, x)=d t^{2}+e^{-2 \lambda t} d x^{2}
$$

where $d t^{2}$ and $d x^{2}$ are the metrics on $\mathbb{R}$ and $\mathfrak{m}_{2}$ induced by the scalar products. In particular the metric on $M$ is a warped product and does not depend on $\omega$. The curvature of $g$ is readily computed to be $-\lambda^{2}$.

To see this, we choose vector fields $T=\frac{\partial}{\partial t}$ tangential to the $\mathbb{R}$-factor and $X, Y$ along the $\mathfrak{m}_{2}$-factor, which are parallel with respect to the product metric $g_{0}=d t^{2}+d x^{2}$. We may also assume that $X$ and $Y$ are perpendicular with respect to both metrics simultaneously. We abbreviate $f(t)=e^{-\lambda t}$ and write $|X|$ for the norm of $X$ with respect to the product metric $g_{0}$. We have

$$
g(T, T)=1, \quad g(X, T)=0=g(X, Y), \quad g(X, X)=f^{2}|X|^{2} .
$$


The various covariant derivatives are

$$
\nabla_{T} T,=0 \quad \nabla_{T} X=\frac{f^{\prime}}{f} X, \quad \nabla_{X} X=-f^{\prime} f|X|^{2} T, \quad \nabla_{X} Y=0 .
$$

Hence, since the vector fields $T, X, Y$ commute,

$$
\begin{aligned}
K(X+T, Y) & =g\left(R_{X+T, Y} Y, X+T\right)=g\left(\nabla_{X+T} \nabla_{Y} Y-\nabla_{Y} \nabla_{X+T} Y, X+T\right) \\
& =g\left(-|Y|^{2} \nabla_{X+T} f^{\prime} f T-\frac{f^{\prime}}{f} \nabla_{Y} Y, X+T\right) \\
& =g\left(-|Y|^{2} \frac{f^{\prime}}{f} f^{\prime} f X-|Y|^{2} f^{\prime \prime} f T-|Y|^{2} f^{\prime 2} T+\frac{f^{\prime}}{f} f^{\prime} f|Y|^{2} T, X+T\right) \\
& =-|Y|^{2}|X|^{2} f^{\prime 2} f^{2}-|Y|^{2} f^{\prime \prime} f-|Y|^{2} f^{\prime 2}+f^{\prime 2}|Y|^{2}
\end{aligned}
$$

For $f(t)=e^{-\lambda t}$ this becomes

$$
-\lambda^{2}\left(|Y|^{2}|X|^{2} f^{4}+|Y|^{2} f^{2}\right)=-\lambda^{2}|(X+T) \wedge Y|^{2}
$$

hence the sectional curvature of the plane spanned by $X+T$ and $Y$ is $-\lambda^{2}$. Since the set of planes of this type is dense, the sectional curvature of $M$ is constant $-\lambda^{2}$.

\section{The Classification IN THE HOMOgEnEOUS CASE}

In this section, we will classify all simply connected Riemannian homogeneous spaces whose isotropy action is of cohomogeneity two. Let $M$ be a simply connected Riemannian homogeneous space and $G$ the connected component of the isometry group of $M$. We denote by $K=G_{p}$ be the isotropy subgroup at a point $p \in M$. Then $K$ is a compact subgroup of $G$ and since $M=G / K$ is simply connected it follows that $K$ is connected. Let $\mathfrak{m}=T_{p} M$ be the tangent space of $M$ at the point $p$ with the scalar product $\langle\cdot \mid \cdot\rangle$ induced by the Riemannian metric of $M$. The action of $K$ on $\mathfrak{m}$ by $\left.\operatorname{Ad}_{G}\right|_{K}$ is orthogonal with respect to this scalar product.

We assume that the isotropy representation of $K$ on $\mathfrak{m}$ is of cohomogeneity two, thus it is either irreducible or has two summands $\mathfrak{m}=\mathfrak{m}_{1} \oplus \mathfrak{m}_{2}$ both of cohomogeneity one. We start with the case where $\mathfrak{m}$ is irreducible.

4.1. Isotropy irreducible spaces. Assume $M=G / K$ is isotropy irreducible, i.e. $K$ acts irreducibly on the tangent space $T_{p} M$. In case $M$ is non-compact, the space is symmetric by [11]. Now assume that $M$ is compact and has isotropy representation of cohomogeneity two. By a result in [11, such a compact $M$ is symmetric or $G$ is simple and compact. Since a cohomogeneity two representation is polar, it follows from [7] that $M$ is symmetric and we have proved:

Theorem 4.1. An isotropy irreducible simply connected homogenous Riemannian manifold $M=G / K$ with isotropy representation of cohomogeneity two is a symmetric space. 
4.2. Reducible isotropy representation. Now assume $M=G / K$ is a simply connected Riemannian homogeneous space whose isotropy representation

$$
T_{p} M=\mathfrak{m}=\mathfrak{m}_{1} \oplus \mathfrak{m}_{2}
$$

consists of two irreducible cohomogeneity one summands $\mathfrak{m}_{1}$ and $\mathfrak{m}_{2}$, such that $K$ acts transitively on $\boldsymbol{S m}_{1} \times \mathbf{S m}_{2}$, where $\mathbf{S m}_{i}$ denotes the unit sphere in $\mathfrak{m}_{i}$.

Let $N_{i}$ be the kernel of $K \rightarrow \mathrm{SO}\left(\mathfrak{m}_{i}\right)$. The fixed modules of $N_{1}$ on $\mathfrak{m}_{2}$ and of $N_{2}$ on $\mathfrak{m}_{1}$ are $K$-invariant subspaces and therefore trivial by effectivity. If both $N_{1}$ and $N_{2}$ are nontrivial, then $M$ splits by Corollary 2.4. But we may assume that $M$ is de Rham irreducible, since otherwise $M$ is just the Riemannian product of two manifolds both of which are two-point homogeneous. We conclude that $K$ acts effectively on at least one of $\mathfrak{m}_{1}$ or $\mathfrak{m}_{2}$, say on $\mathfrak{m}_{2}$.

We will now determine all reducible compact Lie group representations of cohomogeneity two without trivial submodules and where at least one submodule is effective.

Proposition 4.2. Let $K$ be a compact Lie group and let $\mathfrak{m}=\mathfrak{m}_{1} \oplus \mathfrak{m}_{2}$ be an orthogonal $K$-representation such that:

(i) $\mathfrak{m}_{1}$ and $\mathfrak{m}_{2}$ are invariant subspaces,

(ii) $K$ acts nontrivially on $\mathfrak{m}_{1}$;

(iii) $K$ acts effectively on $\mathfrak{m}_{2}$;

(iv) the action of $K$ on $\mathfrak{m}$ is of cohomogeneity two.

Then $K, \mathfrak{m}_{1}$ and $\mathfrak{m}_{2}$ are as given in Table 1 .

\begin{tabular}{|c|c|c|c|c|}
\hline No. & $K$ & $\operatorname{dim} \mathfrak{m}_{1}$ & $\operatorname{dim} \mathfrak{m}_{2}$ & Range \\
\hline \hline$(\mathbf{1})$ & $\mathrm{U}(n)$ & 2 & $2 n$ & $n \geq 3$ \\
\hline $\mathbf{( 2 )}$ & $\mathrm{U}(1) \cdot \operatorname{Sp}(n)$ & 2 & $4 n$ & $n \geq 1$ \\
\hline $\mathbf{( 3 )}$ & $\mathrm{Sp}(1) \cdot \operatorname{Sp}(n)$ & 3 & $4 n$ & $n \geq 1$ \\
\hline $\mathbf{( 4 )}$ & $\mathrm{Spin}(6)=\mathrm{SU}(4)$ & 6 & 8 & \\
\hline $\mathbf{( 5 )}$ & $\operatorname{Spin}(7)$ & 7 & 8 & \\
\hline
\end{tabular}

TABLE 1. Reducible representations of cohomogeneity two

Remark 4.3. While for the entries (3), (4) and (5) of Table 1 the representations are determined up to equivalence by the dimensions of the irreducible modules $\mathfrak{m}_{1}$ and $\mathfrak{m}_{2}$, the table entries (1) and (2) stand for infinitely many equivalence classes of representations, depending on the action of the abelian factor on the module $\mathfrak{m}_{1}$. In case of representation (1), the action of $g \in \mathrm{U}(n)$ on $\mathfrak{m}_{1}=\mathbb{C}$ is given by multiplication with a power of the determinant $\operatorname{det} g^{k}$, where we may assume that $k$ is a positive integer, since the representations of $K$ by $\operatorname{det} g^{k}$ and $\operatorname{det} g^{-k}$ on $\mathfrak{m}_{1}$ (considered to be a real vector space) are equivalent. Similarly, in case of representation (2), the action of $\mathrm{U}(1) \cdot \operatorname{Sp}(n)=\{z A|z \in \mathbb{C}| z \mid,=1, A \in \operatorname{Sp}(n)\}$ on $\mathfrak{m}_{1}=\mathbb{C}$ is given by multiplication with $z^{2 k}$, where $k$ is a positive integer.

We may impose the condition $n \geq 3$ on representation (1) since for $n=1$ the cohomogeneity is 3 and for $n=2$ the action is equivalent to (2). 
Proof. To prove the proposition, one may use the well known classification of Lie groups acting transitively and effectively on spheres by isometries; see e.g. [3], 7.13. For the convenience of the reader, we reproduce it here in Table 2. It is straightforward to find for

\begin{tabular}{|c|c|c|}
\hline Group $K$ & Isotropy $K_{q}$ & $\operatorname{dim} \mathfrak{m}_{2}$ \\
\hline \hline $\mathrm{SO}(n)$ & $\mathrm{SO}(n-1)$ & $n$ \\
\hline $\mathrm{SU}(n)$ & $\mathrm{SU}(n-1)$ & $2 n$ \\
\hline $\mathrm{Sp}(n)$ & $\mathrm{Sp}(n-1)$ & $4 n$ \\
\hline $\mathrm{U}(n)$ & $\mathrm{U}(n-1)$ & $2 n$ \\
\hline $\mathrm{Sp}(n) \cdot \mathrm{Sp}(1)$ & $\mathrm{Sp}(n-1) \cdot \mathrm{Sp}(1)$ & $4 n$ \\
\hline $\mathrm{Sp}(n) \cdot \mathrm{U}(1)$ & $\mathrm{Sp}(n-1) \cdot \mathrm{U}(1)$ & $4 n$ \\
\hline $\mathrm{G}_{2}$ & $\mathrm{SU}(3)$ & 7 \\
\hline $\mathrm{Spin}(7)$ & $\mathrm{G}_{2}$ & 8 \\
\hline $\mathrm{Spin}(9)$ & $\mathrm{Spin}(7)$ & 16 \\
\hline
\end{tabular}

TABLE 2. Representations of cohomogeneity one, $n>1$

any such representation $\mathfrak{m}_{2}$ of a compact Lie group $K$ all other (not necessarily effective) $K$-representations $\mathfrak{m}_{1}$ of cohomogeneity one such that the action of $K$ on $\mathfrak{m}_{1} \oplus \mathfrak{m}_{2}$ is of cohomogeneity two, i.e. the action of $K$ on $\mathfrak{m}_{1}$ restricted to a principal isotropy group of the $K$-action on $\mathfrak{m}_{2}$ still acts with cohomogeneity one.

Alternatively, one may start with the classification of reducible cohomogeneity two representations given in [10] or [2] and then exclude those representations where both modules are non-effective.

Let $\mathfrak{g}$ be the Lie algebra of $G$ and let $\mathfrak{k}$ be the subalgebra corresponding to $K$. Then $\mathfrak{g}$ is of the form

$$
\mathfrak{g}=\mathfrak{k} \oplus \mathfrak{m}_{1} \oplus \mathfrak{m}_{2},
$$

where $\mathfrak{m}=\mathfrak{m}_{1} \oplus \mathfrak{m}_{2}$ is an $\left.\operatorname{Ad}_{G}\right|_{K}$-invariant complement of $\mathfrak{k}$ in $\mathfrak{g}$ such that the action of $K$ on $\mathfrak{m}$ is of cohomogeneity two, $\mathfrak{m}_{1}$ and $\mathfrak{m}_{2}$ are invariant subspaces and $\mathfrak{m}_{2}$ is an effective $K$-representation.

In the following we will determine all possible Lie algebra structures on $\mathfrak{g}$. Since we have already fixed the Lie bracket on $\mathfrak{k} \times \mathfrak{k}$ and on $\mathfrak{k} \times \mathfrak{m}$, it remains to determine the set of all skew symmetric maps $[\cdot, \cdot]: \mathfrak{m} \times \mathfrak{m} \rightarrow \mathfrak{g}$ such that the bracket thus defined on $\mathfrak{g} \times \mathfrak{g}$ satisfies the Jacobi identity.

One major restriction for the possible Lie algebra structure comes from representation theory, namely from the fact that any Lie bracket on $\mathfrak{g}$ defines a $K$-equivariant map $\Lambda^{2} \mathfrak{g} \rightarrow \mathfrak{g}$, since the elements of $K$ act on $\mathfrak{g}$ as Lie algebra automorphisms.

We distinguish three cases: The case where $\mathfrak{m}_{1}$ is a trivial one-dimensional submodule is treated in Section 4.5. The case where $\mathfrak{m}$ is equivalent to representation $(\mathbf{1})$ from Table 1 is studied in Section 4.4. We begin with the remaining four representations $[2),(3)$, (4) and (5) from Table 1. 
4.3. Heisenberg isotropy. In this section, we give a classification of all real Lie algebras $\mathfrak{g}$ of simply connected Lie groups $G$ which have a compact subgroup $K$ such that the isotropy representation of the homogeneous space $G / K$ is of cohomogeneity two, without a trivial submodule and equivalent to the isotropy representation of a generalized Heisenberg group endowed with a left invariant metric. Some generalized Heisenberg algebras occur as special cases of this construction.

Proposition 4.4. Let $M=G / K$ be a simply connected Riemannian homogeneous space which is not de Rham decomposable and such that the action of $K$ on the tangent space of $M$ is equivalent to one of the representations (2), (3), (4) or (5). Then $M$ is isometric to one of the homogeneous spaces as given in Table 3 equipped with a G-invariant metric.

\begin{tabular}{|c|c|c|c|c|c|c|}
\hline No. & $K$ & $\operatorname{dim} \mathfrak{m}_{1}$ & $\operatorname{dim} \mathfrak{m}_{2}$ & $F$ & $B$ & $M$ \\
\hline$(2)$ & $\begin{aligned} & \mathrm{U}(1) \cdot \operatorname{Sp}(n) \\
= & \operatorname{Spin}(2) \cdot \operatorname{Sp}(n)\end{aligned}$ & 2 & $4 n$ & $\begin{array}{l}\mathbb{R}^{2} \\
S^{2} \\
S^{2} \\
S^{2}\end{array}$ & $\begin{array}{l}\mathbb{R}^{4 n} \\
\mathbb{H P}^{n} \\
\mathbb{R}^{4 n} \\
\mathbb{H} H^{n}\end{array}$ & $\begin{array}{c}N(2, n) \\
\frac{\operatorname{Sp}(n+1)}{\mathrm{U}(1) \cdot \operatorname{Sp}(n)} \\
\mathrm{Sp}(1) \cdot \operatorname{Sp}(n) \ltimes \mathbb{R}^{4 n} \\
\mathrm{U}(1) \cdot \operatorname{Sp}(n) \\
\frac{\mathrm{Sp}(n, 1)}{\mathrm{U}(1) \cdot \operatorname{Sp}(n)}\end{array}$ \\
\hline$(3)$ & $\begin{aligned} & \operatorname{Sp}(1) \cdot \operatorname{Sp}(n) \\
= & \operatorname{Spin}(3) \cdot \operatorname{Sp}(n)\end{aligned}$ & 3 & $4 n$ & $\begin{array}{l}\mathbb{R}^{3} \\
S^{3} \\
S^{3} \\
S^{3}\end{array}$ & $\begin{array}{l}\mathbb{R}^{4 n} \\
\mathbb{H P}^{n} \\
\mathbb{R}^{4 n} \\
\mathbb{H} H^{n}\end{array}$ & $\begin{array}{c}N(3 ; n, 0) \\
\frac{\operatorname{Sp}(1) \cdot \operatorname{Sp}(n+1)}{\Delta \operatorname{Sp}(1) \cdot \operatorname{Sp}(n} \\
\frac{\operatorname{Sp}(1) \cdot\left(\operatorname{Sp}(1) \cdot \operatorname{Sp}(n) \ltimes \mathbb{R}^{4 n}\right)}{\Delta \operatorname{Sp}(1) \cdot \operatorname{Sp}(n)} \\
\frac{\operatorname{Sp}(1) \cdot \operatorname{Sp}(n, 1)}{\Delta \operatorname{Sp}(1) \cdot \operatorname{Sp}(n)}\end{array}$ \\
\hline$(4)$ & $\mathrm{SU}(4)=\operatorname{Spin}(6)$ & 6 & 8 & $\begin{array}{l}\mathbb{R}^{6} \\
S^{6}\end{array}$ & $\begin{array}{l}\mathbb{R}^{8} \\
\mathbb{R}^{8}\end{array}$ & $\begin{array}{c}N(6,1) \\
\frac{\operatorname{Spin}(7) \ltimes \delta_{7}}{\operatorname{Spin}(6)}\end{array}$ \\
\hline$(5)$ & $\operatorname{Spin}(7)$ & 7 & 8 & $\begin{array}{l}\mathbb{R}^{7} \\
S^{7} \\
S^{7} \\
S^{7}\end{array}$ & $\begin{array}{l}\mathbb{R}^{8} \\
\mathrm{~S}^{8} \\
\mathbb{R}^{8} \\
\mathrm{H}^{8}\end{array}$ & $\begin{array}{c}N(7 ; 1,0) \\
\frac{\operatorname{Spin}(9)}{\operatorname{Spin}(7)} \\
\frac{\operatorname{Spin}(8) \ltimes \delta_{8}^{+}}{\operatorname{Spin}(7)} \\
\frac{\operatorname{Spin}(8,1)}{\operatorname{Spin}(7)}\end{array}$ \\
\hline
\end{tabular}

TABLE 3. Non-symmetric homogeneous spaces whose isotropy representation is of cohomogeneity two without a trivial submodule

Proof. Let $\mathfrak{g}=\mathfrak{k} \oplus \mathfrak{m}_{1} \oplus \mathfrak{m}_{2}$ be a real Lie algebra and $G$ be the corresponding simply connected Lie group such that the action of the connected subgroup $K$ corresponding to the 
Lie algebra $\mathfrak{k}$ is equivalent to one of the representations $(\mathbf{2 )}, \mathbf{( 3 )}, \mathbf{( 4 )}$, or (5) given in Table 1 . Assume the homogenous space $M=G / K$ is endowed with a $G$-invariant Riemannian metric.

In each case the group $K$ is of the form $K=K_{0} \cdot K_{1}$, where $K_{0}=\operatorname{Spin}(n), n=2,3$, 6 , or 7 , respectively; the $K$-module $\mathfrak{m}_{1}=\mathbb{R}^{n}$ is equivalent to the standard representation of $\operatorname{Spin}(n)$. Since there is an element in the center of $K$ which acts trivially on $\mathfrak{m}_{1}$ and as minus identity on $\mathfrak{m}_{2}$, we know that $\mathfrak{g}_{1}=\mathfrak{k} \oplus \mathfrak{m}_{1}$ is a symmetric subalgebra of $\mathfrak{g}$, i.e. a (not necessarily compact) subalgebra of $\mathfrak{g}$ for which the Cartan relations hold; i.e. we have

$$
\left[\mathfrak{g}_{1}, \mathfrak{g}_{1}\right] \subseteq \mathfrak{g}_{1}, \quad\left[\mathfrak{g}_{1}, \mathfrak{m}_{2}\right] \subseteq \mathfrak{m}_{2}, \quad\left[\mathfrak{m}_{2}, \mathfrak{m}_{2}\right] \subseteq \mathfrak{g}_{1}
$$

We start by determining all possible brackets on $\mathfrak{m}_{1} \times \mathfrak{m}_{1}$. Since the action of $K$ on $\mathfrak{m}_{1}$ is the standard representation of $\operatorname{Spin}(n)$, the $K$-module $\Lambda^{2} \mathfrak{m}_{1}$ is equivalent to the adjoint representation of $K_{0}$. Hence we have by Schur's Lemma that

$$
\left[\mathfrak{m}_{1}, \mathfrak{m}_{1}\right] \subseteq \mathfrak{k}_{0}
$$

and it follows that the space of $K$-equivariant maps $\Lambda^{2} \mathfrak{m}_{1}$ is real one-dimensional, since the adjoint representation of $\mathfrak{k}_{0}$ is of real type.

We shall now explicitly describe the Lie bracket on the subalgebra $\mathfrak{g}_{1}=\mathfrak{k} \oplus \mathfrak{m}_{1}$ of $\mathfrak{g}$. Let $e_{1}, \ldots, e_{n}$ be an orthonormal basis of $\mathfrak{m}_{1}$. Let $\mathrm{C} \ell_{n}=\mathrm{C} \ell\left(\mathfrak{m}_{1}\right)$ be the Clifford algebra over $\mathfrak{m}_{1}$, i.e. the real algebra generated by $e_{1}, \ldots, e_{n}$ subject to the relation $e_{i} \cdot e_{j}+e_{j} \cdot e_{i}=-2 \delta_{i j}$.

Identify $\mathfrak{k}_{0}$ with the subset

$$
\mathfrak{s p i n}(n)=\operatorname{span}_{\mathbb{R}}\left\{e_{i} \cdot e_{j} \mid 1 \leq i<j \leq n\right\} \subset \mathrm{C} \ell_{n},
$$

where the bracket is given by the commutator. Then the action of $\mathfrak{k}_{0}$ on $\mathfrak{m}_{1}$ can be described by $\left[e_{i} \cdot e_{j}, e_{k}\right]=-e_{k} \cdot e_{i} \cdot e_{j}$. Now let $\lambda \in \mathbb{R}$ be a constant. We define a Lie bracket on $\mathfrak{m}_{1} \times \mathfrak{m}_{1}$ by

$$
\left[e_{i}, e_{j}\right]=\lambda e_{i} \cdot e_{j} \in \mathfrak{k}_{0}, i \neq j .
$$

It can be easily verified that the bracket defined in this way is a skew symmetric map on $\mathfrak{g}_{1} \times \mathfrak{g}_{1} \rightarrow \mathfrak{g}_{1}$ fulfilling the Jacobi identity and indeed we have

$$
\mathfrak{g}_{0}=\mathfrak{k}_{0} \oplus \mathfrak{m}_{1} \cong \begin{cases}\mathfrak{s p i n}(n+1) & \text { if } \lambda>0 ; \\ \mathfrak{s p i n}(n) \ltimes \mathbb{R}^{n} & \text { if } \lambda=0 ; \\ \mathfrak{s p i n}(n, 1) & \text { if } \lambda<0\end{cases}
$$

Since the space of $K$-equivariant maps $\Lambda^{2} \mathfrak{m}_{1} \rightarrow \mathfrak{k}_{0}$ is real one-dimensional and $K_{1}$ acts trivially on $\mathfrak{m}_{1}$, this construction exhausts all possible Lie algebra structures on $\mathfrak{g}_{1}=\mathfrak{k} \oplus \mathfrak{m}_{1}$. We have thus shown that the Lie bracket on $\mathfrak{m}_{1} \times \mathfrak{m}_{1}$ is given by (4.7) for some real constant $\lambda$.

Let us now determine the Lie bracket on $\mathfrak{m}_{1} \times \mathfrak{m}_{2}$. From (4.5) it follows that

$$
\left[\mathfrak{m}_{1}, \mathfrak{m}_{2}\right] \subseteq \mathfrak{m}_{2}
$$

Since we consider $K$-representations where the group $K$ acts on $\mathfrak{m}_{1} \oplus \mathfrak{m}_{2}$ as the automorphism group of a Clifford module of the Clifford algebra $\mathrm{C} \ell_{n}$ (cf. [9] or Propositions 3.2 and 3.3 
of [8]), we may assume that $\mathfrak{m}_{2}$ carries the structure of a Clifford module over $\mathrm{C} \ell_{n}$ such that the action of $\mathfrak{k}_{0}$ on $\mathfrak{m}_{2}$ is given by Clifford multiplication, i.e.

$$
[X, w]=X \cdot w, \quad \text { for } X \in \mathfrak{s p i n}(n), w \in \mathfrak{m}_{2},
$$

where we use the identification (4.6). Let us determine the space of $K$-equivariant maps $\mathfrak{m}_{1} \times \mathfrak{m}_{2} \rightarrow \mathfrak{m}_{2}$.

We shall prove that $\operatorname{Hom}_{K}\left(\mathfrak{m}_{1} \otimes \mathfrak{m}_{2}, \mathfrak{m}_{2}\right)$ has real dimension at most one in the case of representations (3) and (5) and complex dimension at most one in case of representations (2) and (4).

For the representations (2), where the action of $z A, z \in \mathbb{C},|z|=1, A \in \operatorname{Sp}(n)$ on $\mathfrak{m}_{1}=\mathbb{C}$ is given by complex multiplication with $z^{2 k}$, the real $K$-module $\mathfrak{m}_{1} \otimes \mathfrak{m}_{2}$ decomposes into two $4 n$-dimensional submodules; on these two submodules, the action of $z A$ is given by the matrices $z^{2 k+1} A$ and $z^{2 k-1} A$, respectively. Thus it follows that $\mathfrak{m}_{1} \otimes \mathfrak{m}_{2}$ contains a submodule equivalent to $\mathfrak{m}_{2}$ if and only if $k=1$; furthermore, $\mathfrak{m}_{1} \otimes \mathfrak{m}_{2}$ contains at most one such submodule.

In case of the representations (3) and (5), the real $K$-representation $\mathfrak{m}_{1} \otimes \mathfrak{m}_{2}$ contains at most one submodule equivalent to $\mathfrak{m}_{2}$. (To verify this, one may for example use Weyl's dimension formula to see that the tensor product of the standard and the spin representation of $\operatorname{Spin}(3)$ or Spin(7) contains an irreducible module of real dimension 8 or 48, respectively.) Since $\mathfrak{m}_{2}$ is a $K$-representation of real type, it follows that the space of $K$-equivariant maps $\mathfrak{m}_{1} \times \mathfrak{m}_{2} \rightarrow \mathfrak{m}_{2}$ is at most real one-dimensional.

Let us now consider the case of representation (4). Here the real $K$-modules $\mathfrak{m}_{1}$ and $\mathfrak{m}_{2}$ are of real and complex type, respectively, and hence we may view the $K$-representation $\mathfrak{m}_{1} \otimes \mathfrak{m}_{2}$ as a complex $K$-module. The real tensor product $\mathfrak{m}_{1} \otimes \mathfrak{m}_{2}$ decomposes into a complex 20-dimensional irreducible plus a complex 4-dimensional module; thus the space of $K$-equivariant maps $\mathfrak{m}_{1} \times \mathfrak{m}_{2} \rightarrow \mathfrak{m}_{2}$ is at most complex one-dimensional.

Clifford multiplication induces a map

$$
\mathfrak{m}_{1} \times \mathfrak{m}_{2} \rightarrow \mathfrak{m}_{2}, \quad(v, w) \mapsto v \cdot w
$$

which is $K$-equivariant since $K$ acts on $\mathfrak{m}_{1} \oplus \mathfrak{m}_{2}$ as a group of Clifford module automorphisms, cf. [9]. We have shown that the Lie bracket on $\mathfrak{m}_{1} \times \mathfrak{m}_{2}$ is given by

$$
[v, w]=\mu v \cdot w
$$

where $\mu$ is a real constant in case of representations (3) or (5) and a complex constant in case of representations (2) or (4). Let $X, Y \in \mathfrak{m}_{1}, Z \in \mathfrak{m}_{2}$. By using (4.7) and (4.8), it follows from the Jacobi identity $[[X, Y], Z]=[X,[Y, Z]]-[Y,[X, Z]]$ that $\lambda=2 \mu^{2}$.

We can always rescale the Lie bracket to have $\lambda=0$ or \pm 1 and furthermore change the sign of $\mu$. In fact, consider a linear map $f: \mathfrak{k} \oplus \mathfrak{m}_{1} \oplus \mathfrak{m}_{2} \rightarrow \mathfrak{k} \oplus \mathfrak{m}_{1} \oplus \mathfrak{m}_{2}$ mapping $(k, x, v) \mapsto(k, \alpha x, v)$, and denote by $[\cdot, \cdot]^{\prime}$ the Lie bracket pulled back via $f$, i.e. $[x, y]^{\prime}=f^{-1}[f x, f y]$. For $y \in \mathfrak{m}_{2}$ and $u, v \in \mathfrak{m}_{1}$ we then have

$$
\begin{aligned}
& {[u, v]^{\prime}=f^{-1}[f u, f v]=\lambda f u \cdot f v=\alpha^{2} \lambda[u, v]^{\prime} \in \mathfrak{k} \quad \text { and }} \\
& {[u, y]^{\prime}=f^{-1}[f u, f y]=f^{-1}(\mu f u \cdot f y)=\alpha \mu[u, y]^{\prime} \in \mathfrak{m}_{2} .}
\end{aligned}
$$


We can thus rescale $(\lambda, \mu)$ to $\left(\alpha^{2} \lambda, \alpha \mu\right)$.

We will now consider the Lie bracket on $\mathfrak{m}_{2} \times \mathfrak{m}_{2}$. From (4.5) we know that

$$
\left[\mathfrak{m}_{2}, \mathfrak{m}_{2}\right] \subseteq \mathfrak{k} \oplus \mathfrak{m}_{1}
$$

To proceed further, we distinguish between two cases, depending on whether the modules $\mathfrak{m}_{1}$ and $\mathfrak{m}_{2}$ do or do not commute.

The case where $\mathfrak{m}_{1}$ and $\mathfrak{m}_{2}$ commute. Assume $\left[\mathfrak{m}_{1}, \mathfrak{m}_{2}\right]=0$. Then we have $\lambda=2 \mu^{2}=0$ and it follows that $\mathfrak{m}_{1}$ is an abelian ideal of $\mathfrak{g}$.

If in addition $\left[\mathfrak{m}_{2}, \mathfrak{m}_{2}\right]=0$ holds, then $\mathfrak{m}=\mathfrak{m}_{1} \oplus \mathfrak{m}_{2}$ is an abelian ideal of $\mathfrak{g}$ and $M=G / K$ is a homogeneous presentation of a Euclidean space.

Now assume $0 \neq\left[\mathfrak{m}_{2}, \mathfrak{m}_{2}\right] \subseteq \mathfrak{m}_{1}$; in this case, the ideal $\mathfrak{m}_{1} \oplus \mathfrak{m}_{2}$ of $\mathfrak{g}$ is a two-step nilpotent Lie algebra with center $\mathfrak{m}_{1}$. The Lie bracket on $\mathfrak{m}_{2} \times \mathfrak{m}_{2}$ defines a $K$-equivariant map $Z \mapsto J_{Z}$ from $\mathfrak{m}_{1}$ to the skew symmetric endomorphisms of $\mathfrak{m}_{2}$ by $\left\langle J_{Z} X \mid Y\right\rangle=\langle Z \mid[X, Y]\rangle$ and also a $K$-equivariant map $\varphi: \mathfrak{m}_{1} \times \mathfrak{m}_{2} \rightarrow \mathfrak{m}_{2}$ by

$$
\langle\varphi(Z, X) \mid Y\rangle=\langle Z \mid[X, Y]\rangle=\left\langle J_{Z} X \mid Y\right\rangle \quad \text { for } Z \in \mathfrak{m}_{1}, X, Y \in \mathfrak{m}_{2} .
$$

We have already shown that the space of $K$-equivariant maps $\mathfrak{m}_{1} \times \mathfrak{m}_{2} \rightarrow \mathfrak{m}_{2}$ is real onedimensional for representations (3) or (5) and complex one-dimensional in case of representations (2) or (4). One particular such $K$-equivariant map is given by Clifford multiplication using the inclusion $\mathfrak{m}_{1} \subset \mathrm{C} \ell\left(\mathfrak{m}_{1}\right)$. It follows that the map $J: \mathfrak{m}_{1} \rightarrow \operatorname{End}\left(\mathfrak{m}_{2}\right), Z \mapsto J_{Z}$ is given by $J_{Z}: X \mapsto \kappa Z \cdot X$ where $\kappa$ is a real constant in case of representations (3) or (5) and a complex constant in case of representations (2) or (4), the dot denoting Clifford multiplication. (In case of representations (2) and (4), $\mathfrak{m}_{2}$ carries a complex structure.) Since we assume $\left[\mathfrak{m}_{2}, \mathfrak{m}_{2}\right] \neq 0$, it follows that $\kappa \neq 0$.

Now let $\rho=\sqrt{|\kappa|}, \varepsilon=\operatorname{sgn}(\kappa)$ in case of representations $(3)$ or (5) and let $\rho \in \mathbb{C}$ be such that $\rho^{2}=\kappa$ and $\varepsilon=+1$, in case of representations (2) or (4). Define a bijective linear map $f: \mathfrak{m} \rightarrow \mathfrak{m}$ by $f(Z)=\varepsilon Z$ for $Z \in \mathfrak{m}_{1} ; f(X)=X / \rho$ for $X \in \mathfrak{m}_{1}$. It follows that $\langle f(Z) \mid[f(X), f(Y)]\rangle=\left\langle J_{f(Z)} f(X) \mid f(Y)\right\rangle=\langle Z \cdot X \mid Y\rangle$. This shows that $\mathfrak{m}$ is isomorphic to the Lie algebra of a generalized Heisenberg group of type

$$
N(2,1), N(3 ; 1,0), N(6,1) \text {, or } N(7 ; 1,0),
$$

cf. [1] 3.1, and that $M$ endowed with the metric induced from the scalar product $\langle\cdot \mid \cdot\rangle$ is isometric to a generalized Heisenberg group. We have shown that all possible two-step nilpotent Lie algebra structures on $\mathfrak{m}$ are given by this construction.

Now assume $\left[\mathfrak{m}_{2}, \mathfrak{m}_{2}\right] \nsubseteq \mathfrak{m}_{1}$. Since $\mathfrak{m}_{1}$ is an (abelian) ideal, we may consider the quotient algebra $\mathfrak{g} / \mathfrak{m}_{1}$, of which $\mathfrak{k}$ is a subalgebra via $\mathfrak{k} \rightarrow \mathfrak{g} / \mathfrak{m}_{1}: X \mapsto X+\mathfrak{m}_{1}$. From (4.5) we have that $\mathfrak{k}$ is a compact symmetric subalgebra of $\mathfrak{g} / \mathfrak{m}_{1}$. In case of representations (2), (4)] and (5) this leads to a contradiction with the classification of symmetric spaces [5], since in these cases the $K$-representation on $\mathfrak{m}_{2}$ is not equivalent to an isotropy representation of any Riemannian symmetric space. For representation $(3)$, it follows that $\mathfrak{g} / \mathfrak{m}_{1}$ is a simple Lie algebra of type $\mathfrak{s p}(n+1)$ or $\mathfrak{s p}(n, 1)$. But $\mathfrak{g} / \mathfrak{m}_{1}$ acts nontrivially with nontrivial kernel $\mathfrak{k}_{1} \oplus \mathfrak{m}_{2}$ on $\mathfrak{m}_{1}$ and hence cannot be simple. 
The case where $\mathfrak{m}_{1}$ and $\mathfrak{m}_{2}$ do not commute. In this case we have $\lambda=2 \mu^{2} \neq 0$. Furthermore, since $\mathfrak{m}_{2}$ is $K$-irreducible, we have $\left[\mathfrak{m}_{1}, \mathfrak{m}_{2}\right]=\mathfrak{m}_{2}$ from (4.5). Let $G_{1}$ be the connected subgroup of $G$ corresponding to $\mathfrak{g}_{1}$, which is closed by Theorem 3.1. Up to scaling, the homogeneous space $F=G_{1} / K$ is (locally) isometric to either $\mathrm{H}^{n}$, in case $\lambda<0$, or $\mathrm{S}^{n}$, in case $\lambda>0$. By Theorem 3.4 it follows that the action of $G_{1}$ on $\mathfrak{m}_{2}$ is isometric.

If $\lambda<0$, it follows that $\mathfrak{k}_{0} \oplus \mathfrak{m}_{1} \cong \mathfrak{s p i n}(2,1), \mathfrak{s p i n}(3,1), \mathfrak{s p i n}(6,1)$, or $\mathfrak{s p i n}(7,1)$; in particular, $\mathfrak{k}_{0} \oplus \mathfrak{m}_{1}$ is a simple real Lie algebra. It follows that the corresponding group acts nontrivially and isometrically on $\mathfrak{m}_{2}$, a contradiction. Thus we may henceforth assume $\lambda>0$. By the rescaling (4.9) we can actually arrange for $\lambda=1$ and $\mu=1 / \sqrt{2}$.

Now assume $\left[\mathfrak{m}_{2}, \mathfrak{m}_{2}\right]=0$, i.e. $\mathfrak{m}_{2}$ is an abelian ideal of $\mathfrak{g}$. Then the Lie bracket of $\mathfrak{g}$ is uniquely determined by $\lambda$ and $\mu$. Hence it suffices to exhibit examples of such spaces $M$ in order to explicitly obtain the Lie algebra structure (up to rescaling) and we conclude that $M=G / K$ is isometric to one of the homogeneous spaces

$$
\frac{\operatorname{Sp}(1) \cdot \operatorname{Sp}(n) \ltimes \mathbb{R}^{4 n}}{\mathrm{U}(1) \cdot \operatorname{Sp}(n)}, \quad \frac{\operatorname{Sp}(1) \cdot\left(\operatorname{Sp}(1) \cdot \operatorname{Sp}(n) \ltimes \mathbb{R}^{4 n}\right)}{\Delta \operatorname{Sp}(1) \cdot \operatorname{Sp}(n)}, \quad \frac{\operatorname{Spin}(7) \ltimes \delta_{7}}{\operatorname{Spin}(6)}, \quad \frac{\operatorname{Spin}(8) \ltimes \delta_{8}^{+}}{\operatorname{Spin}(7)}
$$

endowed with a $G$-invariant metric, where $\delta_{7}$ stands for the 8-dimensional spin representation of $\operatorname{Spin}(7)$ and $\delta_{8}^{+}$denotes an 8 -dimensional half-spin representation of $\operatorname{Spin}(8)$; the " $\Delta$ " indicates that the $\operatorname{Sp}(1)$-factor is diagonally embedded into the two $\operatorname{Sp}(1)$-factors of $G$.

If $0 \neq\left[\mathfrak{m}_{2}, \mathfrak{m}_{2}\right] \subseteq \mathfrak{k}$, then $\mathfrak{k}$ is symmetric in $\mathfrak{k} \oplus \mathfrak{m}_{2}$. This is a contradiction for representations (2), (5) and (4), since in these cases $\mathfrak{m}_{2}$ is not equivalent to the isotropy representation of a Riemannian symmetric space. For representation $(\mathbf{3})$, it follows that $\left[\mathfrak{m}_{2}, \mathfrak{m}_{2}\right]=\mathfrak{k}$, but then we obtain from the Jacobi identity

$$
\left[\left[\mathfrak{m}_{2}, \mathfrak{m}_{2}\right], \mathfrak{m}_{1}\right] \subseteq\left[\mathfrak{m}_{2},\left[\mathfrak{m}_{2}, \mathfrak{m}_{1}\right]\right]=\left[\mathfrak{m}_{2}, \mathfrak{m}_{2}\right]
$$

the contradiction $\mathfrak{m}_{1} \subseteq \mathfrak{k}$.

If $\left[\mathfrak{m}_{2}, \mathfrak{m}_{2}\right]=\mathfrak{m}_{1}$, then (4.11) reduces to $\left[\mathfrak{m}_{1}, \mathfrak{m}_{1}\right] \subseteq \mathfrak{m}_{1}$, which implies $\left[\mathfrak{m}_{1}, \mathfrak{m}_{1}\right]=0$. It follows that $\mathfrak{m}$ is a subalgebra of $\mathfrak{g}$ and since neither of the $K$-irreducible spaces $\mathfrak{m}_{1}$ or $\mathfrak{m}_{2}$ is an ideal of $\mathfrak{m}$, the Lie algebra $\mathfrak{m}$ must be simple. By [5], Corollary II 6.5 , it follows that $\mathfrak{m}$ contains a subalgebra isomorphic to $\mathfrak{k}$ whose action on $\mathfrak{m}$ is equivalent to the $\mathfrak{k}$-action. This is a contradiction since $\mathfrak{m}$ does not contain any $K$-submodule equivalent to the adjoint representation of $K$.

It only remains the case where $\mathfrak{m}_{1} \neq\left[\mathfrak{m}_{2}, \mathfrak{m}_{2}\right] \nsubseteq \mathfrak{k}$. In case $n=6,7$ it follows that $\mathfrak{g}$ is simple, immediately leading to a contradiction in case $n=6$, since there is no simple real Lie algebra of dimension 29; if $n=7$ then $\mathfrak{g}$ is a simple real Lie algebra of dimension 36 containing $\mathfrak{s p \mathfrak { i n }}(7)$ as a subalgebra and it follows that $\mathfrak{g}$ is isomorphic to either $\mathfrak{s p i n}(9)$, $\mathfrak{s p i n}(8,1)$ or $\mathfrak{s p i n}(7,2)$; however, only in the first two cases the isotropy representation can be equivalent to (5), hence

$$
M=\operatorname{Spin}(9) / \operatorname{Spin}(7) \quad \text { or } \quad M=\operatorname{Spin}(8,1) / \operatorname{Spin}(7) .
$$

Now consider the case of representations (2) and (3). The subalgebras $\mathfrak{k}_{0} \oplus \mathfrak{m}_{1}$ and $\mathfrak{k}_{1}$ of $\mathfrak{g}_{1}$ commute and hence are ideals of $\mathfrak{g}_{1}$. Since we have already shown that $\lambda$ is positive, 
we know that $\mathfrak{k}_{0} \oplus \mathfrak{m}_{1}$, and therefore also $\mathfrak{g}_{1}$, is a compact Lie algebra. By (4.5) we have that $\mathfrak{g}_{1}$ is a compact symmetric subalgebra of $\mathfrak{g}$.

In case of representation $(\mathbf{2})$ we have $\mathfrak{g}_{1} \cong \mathfrak{s p}(1) \oplus \mathfrak{s p}(n)$ with $G_{1}$ acting transitively and almost effectively on the unit sphere in $\mathfrak{m}_{2}$ and we obtain from the classification of symmetric spaces [5] that $\mathfrak{g}$ is isomorphic to either $\mathfrak{s p}(n+1)$ or $\mathfrak{s p}(n, 1)$. Hence it follows that

$$
M=\operatorname{Sp}(n+1) / \mathrm{U}(1) \cdot \operatorname{Sp}(n) \quad \text { or } \quad M=\mathrm{Sp}(n, 1) / \mathrm{U}(1) \cdot \operatorname{Sp}(n) .
$$

In case of representation (3), we have that $\mathfrak{k}_{0} \oplus \mathfrak{m}_{1} \cong \mathfrak{s o}(4)$ and hence $\mathfrak{g}_{1} \cong \mathfrak{s p}(1) \oplus \mathfrak{s p}(1) \oplus$ $\mathfrak{s p}(n)$. The compact symmetric subgroup $G_{1}$ acts transitively on the unit sphere in $\mathfrak{m}_{2}$ and such that the action of the $\operatorname{Sp}(n)$-factor is almost effective; it follows from the classification of symmetric spaces [5] that $G / G_{1}$ is a non-effective presentation of $\mathbb{H} \mathrm{H}^{n}$ or $\mathbb{H} \mathrm{P}^{n}$. Hence $\mathfrak{g}$ is isomorphic to a Lie algebra direct $\operatorname{sum} \mathfrak{s p}(1) \oplus \mathfrak{s p}(n+1)$ or $\mathfrak{s p}(1) \oplus \mathfrak{s p}(n, 1)$. It follows that

$$
M=\frac{\operatorname{Sp}(1) \cdot \operatorname{Sp}(n+1)}{\Delta \operatorname{Sp}(1) \cdot \operatorname{Sp}(n)} \quad \text { or } \quad M=\frac{\operatorname{Sp}(1) \cdot \operatorname{Sp}(n, 1)}{\Delta \operatorname{Sp}(1) \cdot \operatorname{Sp}(n)},
$$

where the " $\Delta$ " indicates that the $\operatorname{Sp}(1)$-factor is diagonally embedded into the two simple factors of $G$.

4.4. A Lie algebra construction leading to homogeneous presentations of flat space. In this section we will show that a Riemannian homogeneous space whose isotropy representation is equivalent to (1) from Table 1 is Euclidean.

Proposition 4.12. Let $M=G / K$ be a simply connected Riemannian homogeneous space such that the action of $K=\mathrm{U}(n)$ on the tangent space $\mathfrak{m}=\mathfrak{m}_{1} \oplus \mathfrak{m}_{2}=\mathbb{C} \oplus \mathbb{C}^{n}$ of $M$ is equivalent to representation $\mathbf{( 1 )}$, i.e. $K$ acts by a power of the determinant on $\mathfrak{m}_{1}$ and by the standard representation on $\mathfrak{m}_{2}$. Then $M$ is isometric to Euclidean space.

Proof. It follows from Theorem 3.4 that the action of $G_{1}$ on $\mathfrak{m}_{2}$ is isometric, since the principal isotropy group of the $K$-action on $\mathfrak{m}_{2}$ is isomorphic to $\mathrm{U}(n-1)$. Assume the $G_{1}$-action on $\mathfrak{m}_{2}$ is almost effective, then we have $G_{1} \subseteq \mathrm{SO}\left(\mathfrak{m}_{2}\right)$; however, since $\mathfrak{k}=\mathfrak{u}(n) \subset \mathfrak{s o}(2 n)$ is a maximal subalgebra, it follows that $\mathfrak{g}_{1} \cong \mathfrak{s o}(2 n)$, which leads to contradiction by a dimension count. Thus it follows that the action of $\mathfrak{g}_{1}$ has a two-dimensional kernel, which is an ideal of $\mathfrak{g}_{1}$. Since the only two-dimensional $K$-invariant subspace of $\mathfrak{g}_{1}$ is $\mathfrak{m}_{1}$, this shows that $\left[\mathfrak{m}_{1}, \mathfrak{m}_{2}\right]=0$.

Now observe that $\mathfrak{g}_{1}=\mathfrak{k} \oplus \mathfrak{m}_{1}$ is a subalgebra of $\mathfrak{g}$ by Theorem 3.1. Since $\Lambda^{2} \mathfrak{m}_{1}$ is a one-dimensional trivial $K$-module, it follows from Schur's Lemma that $\left[\mathfrak{m}_{1}, \mathfrak{m}_{1}\right] \subseteq \mathfrak{k}_{0}$, where $\mathfrak{k}_{0}$ is the one-dimensional abelian ideal of $\mathfrak{k}=\mathfrak{u}(n)$. But since we have already shown that $\mathfrak{m}_{1}$ is an ideal of $\mathfrak{g}_{1}$, it follows that $\left[\mathfrak{m}_{1}, \mathfrak{m}_{1}\right]=0$.

Since the homogeneous space $\mathrm{SO}(2 n) / \mathrm{U}(n)=\mathrm{SO}\left(\mathfrak{m}_{2}\right) / K$ is isotropy irreducible [11], it is obvious by a dimension count that the $K$-module $\mathfrak{m}_{1} \otimes \mathfrak{m}_{2}$ does not contain any submodule equivalent to $\mathfrak{m}_{1}$ or $\mathfrak{m}_{2}$. Therefore $\left[\mathfrak{m}_{2}, \mathfrak{m}_{2}\right] \subseteq \mathfrak{k}$. Assume $\left[\mathfrak{m}_{2}, \mathfrak{m}_{2}\right] \neq 0$ and consider the Lie algebra $\mathfrak{k} \oplus \mathfrak{m}_{2}$; it follows from the classification of symmetric spaces [5] that $\mathfrak{k} \oplus \mathfrak{m}_{2}$ is a simple real Lie algebra of type $\mathfrak{s u}(n+1)$ or $\mathfrak{s u}(n, 1)$; however, $\mathfrak{k} \oplus \mathfrak{m}_{2}$ acts on $\mathfrak{m}_{1}$ with nontrivial kernel $\mathfrak{m}_{2}$, a contradiction. 
We have proved that $\mathfrak{m}=\mathfrak{m}_{1} \oplus \mathfrak{m}_{2}$ is an abelian ideal of $\mathfrak{g}$ and hence $M$ is flat.

4.5. Isotropy with a one-dimensional trivial module. To complete the classification in the homogeneous case, it remains to study manifolds $M=G / K$ where the isotropy representation has a one-dimensional trivial submodule.

Proposition 4.13. Let $M=G / K$ be a simply connected Riemannian homogeneous space such that the action of $K$ on the tangent space of $M$ is of cohomogeneity two and leaves a nonzero vector fixed. Assume that $M$ is not de Rham decomposable and not symmetric. Then $M$ is isometric to one of the following homogeneous spaces equipped with a G-invariant metric:

$$
\frac{\mathrm{SU}(n+1)}{\mathrm{SU}(n)}, \quad \frac{\mathrm{SU}(n, 1)}{\mathrm{SU}(n)}, \quad N(1, k) .
$$

Proof. Assume $\mathfrak{m}_{1}$ is a one-dimensional trivial $K$-module and $\mathfrak{m}_{2}$ is an irreducible $K$-representation of cohomogeneity one. Clearly we have $\left[\mathfrak{m}_{1}, \mathfrak{m}_{1}\right]=0$.

The case where $\mathfrak{m}_{1}$ and $\mathfrak{m}_{2}$ commute. Assume $\left[\mathfrak{m}_{1}, \mathfrak{m}_{2}\right]=0$. Then $\mathfrak{m}_{1}$ lies in the center of $\mathfrak{g}$ and is hence an ideal of $\mathfrak{g}$. Consider the quotient algebra $\mathfrak{g}_{0}=\mathfrak{g} / \mathfrak{m}_{1}$. It contains $\mathfrak{k}$ as a subalgebra, acting irreducibly on a $K$-invariant complement, denoted by $\overline{\mathfrak{m}}_{2}$, of $\mathfrak{k}$ in $\mathfrak{g}_{0}$; the action of $\mathfrak{k}$ on $\overline{\mathfrak{m}}_{2}$ is clearly equivalent to the $\mathfrak{k}$-action on $\mathfrak{m}_{2}$, thus the corresponding homogeneous space $M_{0}=G_{0} / K$ is strongly isotropy irreducible, with an isotropy representation of cohomogeneity one. It follows that $M_{0}$ equipped with a $G_{0}$-invariant metric is either isometric to a symmetric space of rank one or Euclidean.

Let $[\cdot, \cdot]_{0}$ be the Lie bracket of $\mathfrak{g}_{0}=\mathfrak{k} \oplus \overline{\mathfrak{m}}_{2}$. Consider the subspace $I=\overline{\mathfrak{m}}_{2}+\left[\overline{\mathfrak{m}}_{2}, \overline{\mathfrak{m}}_{2}\right]_{0}$ of $\mathfrak{g}_{0}$. It follows from the Jacobi identity that $I$ is an ideal of $\mathfrak{g}_{0}$, since

$$
[I, \mathfrak{k}]_{0}=\left[\overline{\mathfrak{m}}_{2}+\left[\overline{\mathfrak{m}}_{2}, \overline{\mathfrak{m}}_{2}\right]_{0}, \mathfrak{k}\right]_{0} \subseteq\left[\overline{\mathfrak{m}}_{2}, \mathfrak{k}\right]_{0}+\left[\overline{\mathfrak{m}}_{2},\left[\overline{\mathfrak{m}}_{2}, \mathfrak{k}\right]_{0}\right]_{0}=I
$$

and

$$
\left[I, \overline{\mathfrak{m}}_{2}\right]_{0} \subseteq\left[\mathfrak{g}_{0}, \overline{\mathfrak{m}}_{2}\right]_{0}=\left[\mathfrak{k}, \overline{\mathfrak{m}}_{2}\right]_{0}+\left[\overline{\mathfrak{m}}_{2}, \overline{\mathfrak{m}}_{2}\right]_{0}=I .
$$

It follows that $\mathfrak{m}_{1}+\mathfrak{m}_{2}+\left[\mathfrak{m}_{2}, \mathfrak{m}_{2}\right]$ is an ideal of $\mathfrak{g}$.

Assume first the ideal $I$ is simple. Then $\mathfrak{m}_{1}+\mathfrak{m}_{2}+\left[\mathfrak{m}_{2}, \mathfrak{m}_{2}\right]$ is a central extension of the simple Lie algebra $I$ and hence isomorphic to the Lie algebra direct sum of $I$ and the one-dimensional abelian Lie algebra $\mathfrak{m}_{1}$ by the Whitehead Lemma. Since $\mathfrak{m}_{1}$ lies in the center of $\mathfrak{g}$, it follows that also $\mathfrak{g}$ is isomorphic to a Lie algebra direct sum of $\mathfrak{g}_{0}$ and a one-dimensional abelian Lie algebra. Hence the homogeneous space $M=G / K$ is de Rham decomposable in this case.

Assume now $I=\overline{\mathfrak{m}}_{2}$, i.e. $\left[\overline{\mathfrak{m}}_{2}, \overline{\mathfrak{m}}_{2}\right] \subseteq \overline{\mathfrak{m}}_{2}$. Then the $K$-irreducible space $I=\overline{\mathfrak{m}}_{2}$ is either an abelian or a simple ideal. We have already treated the case where $I$ is simple, thus we may assume $I$ is abelian. In this case $\mathfrak{m}=\mathfrak{m}_{1} \oplus \mathfrak{m}_{2}$ is a two-step nilpotent ideal of $\mathfrak{g}$ and it follows that the Lie bracket on $\mathfrak{m}$ is given by a $K$-invariant skew-symmetric bilinear form $\omega: \mathfrak{m}_{2} \times \mathfrak{m}_{2} \rightarrow \mathbb{R}$ such that

$$
[X, Y]=\omega(X, Y) v \quad \text { for all } X, Y \in \mathfrak{m}_{2},
$$


where $v \in \mathfrak{m}_{1}$ is some nonzero vector. We may assume $\omega \neq 0$ since otherwise $M$ is flat. The two-form $\omega$ defines a nonzero skew-symmetric $K$-equivariant endomorphism $F$ on $\mathfrak{m}_{2}$ by $\langle F(X) \mid Y\rangle=\omega(X, Y)$. Hence $F^{2}$ is a nonzero symmetric $K$-equivariant endomorphism on $\mathfrak{m}_{2}$ with at least one negative eigenvalue. By Schur's Lemma it follows that $F^{2}=-\lambda \mathrm{id}_{\mathfrak{m}_{2}}$, for some $\lambda>0$. Hence the $K$-module $\mathfrak{m}_{2}$ admits a complex structure defined by $F / \sqrt{\lambda}$. It follows that $M$ is isometric to a generalized Heisenberg group of type

$$
N(1, k), \text { where } k=\frac{1}{2} \operatorname{dim}_{\mathbb{R}} \mathfrak{m}_{2} .
$$

We may now assume that $I \neq \overline{\mathfrak{m}}_{2}$ and that $I$ is not simple. Then $I$ contains a non-trivial proper ideal. Since $\mathfrak{k}$ acts effectively on $\overline{\mathfrak{m}}_{2}$, the only possibility is that $\overline{\mathfrak{m}}_{2}$ is an ideal of $I$, contradicting $I \neq \overline{\mathfrak{m}}_{2}$.

The case where $\mathfrak{m}_{1}$ and $\mathfrak{m}_{2}$ do not commute. In view of Theorem 3.4 we may assume that the $G_{1}$-action on $\mathfrak{m}_{2}$ is isometric. Furthermore, as $\left[\mathfrak{m}_{1}, \mathfrak{m}_{2}\right] \neq 0$ we may assume that the group $G_{1}$ acts almost effectively, i.e. with a discrete kernel $\Gamma \subset G_{1}$, on $\mathfrak{m}_{2}$. In fact, if $\mathfrak{g}_{1}$ had a kernel $\mathfrak{b}$ on $\mathfrak{m}_{2}$ then, by the effectivity of the $K$-action on $\mathfrak{m}_{2}, \mathfrak{b}$ would be another $\operatorname{Ad}_{K}$-invariant one-dimensional complement of $\mathfrak{k}$ in $\mathfrak{g}_{1}$; in this case we could use $\mathfrak{b}$ in place of $\mathfrak{m}_{1}$ and would be in the previous case where $\mathfrak{m}_{1}$ and $\mathfrak{m}_{2}$ commute.

Thus both $K$ and $G_{1} / \Gamma$ act effectively and transitively on the sphere $\mathrm{Sm}_{2}$ in $\mathfrak{m}_{2}$. Therefore both must show up in the classification of such groups. From Table 2, the only cases where a group and a one-dimensional extension of it act effectively and simply transitively on the same sphere are the cases

$$
K=\mathrm{SU}(n), \quad G_{1} / \Gamma=\mathrm{U}(n), \quad \mathfrak{m}_{2}=\mathbb{C}^{n}
$$

and

$$
K=\operatorname{Sp}(n), \quad G_{1} / \Gamma=\mathrm{U}(1) \cdot \operatorname{Sp}(n), \quad \mathfrak{m}_{2}=\mathbb{H}^{n} .
$$

In both cases, the only module isomorphic to $\mathfrak{m}_{1} \otimes \mathfrak{m}_{2} \cong \mathfrak{m}_{2}$ in $\mathfrak{g}=\mathfrak{k} \oplus \mathfrak{m}_{1} \oplus \mathfrak{m}_{2}$ is $\mathfrak{m}_{2}$. Therefore $G_{1}$ acts on $\mathfrak{m}_{2}$ in the usual sense (i.e. the projection (3.2) is not needed). Also, $G_{1}$ contains an element in its center acting as -1 on $\mathfrak{m}_{2}$. Hence the Cartan relations (4.5) hold and $\left(\mathfrak{g}, \mathfrak{g}_{1}\right)$ is an orthogonal symmetric Lie algebra. It follows from the classification of symmetric spaces that $G \cong \mathrm{SU}(n+1)$ or $\cong \mathrm{SU}(n, 1)$ if $\mathfrak{m}_{2}$ is not abelian, hence

$$
M=\mathrm{SU}(n+1) / \mathrm{SU}(n) \quad \text { or } \quad M=\mathrm{SU}(n, 1) / \mathrm{SU}(n) \text {. }
$$

If $\mathfrak{m}_{2}$ is abelian, then $\mathfrak{m}=\mathfrak{m}_{1} \oplus \mathfrak{m}_{2}$ is an ideal of $\mathfrak{g}$. Since the action of $\mathfrak{m}_{1}$ on $\mathfrak{m}_{2}$ commutes with the irreducible $K$-action it follows that $\mathfrak{m}_{2}$ carries a $K$-equivariant complex structure and such that the action of elements in $\mathfrak{m}_{1}$ is given as multiplication by purely imaginary elements of $\mathbb{C}$. It follows as in Theorem 3.4 that the simply connected Lie group corresponding to $\mathfrak{m}$ is isomorphic to the group $M=\mathbb{R} \ltimes \mathbb{C}^{n}$, where the group multiplication is given by

$$
(t, v) \cdot(s, w)=\left(t+s, v+e^{i t} w\right) .
$$

Identifying $M$ with $\mathbb{R}^{1+2 n}$, this induces a simply transitive action of $M$ on itself which is isometric if $M=\mathbb{R}^{1+2 n}$ endowed with any Riemannian metric of the form

$$
d s^{2}=\lambda d x_{1}^{2}+\mu\left(d x_{2}^{2}+\cdots+d x_{1+2 n}^{2}\right),
$$


where $\lambda, \mu>0$ are constants. This shows that, for any choice of a left invariant metric, $M=G / K$ is isometric to Euclidean space, hence de Rham decomposable.

We have now completed the proof of Theorem 1.1 .

\section{The inhomogeneous CASE}

In this section we classify simply connected manifolds admitting a non-transitive group of isometries whose isotropy groups act with cohomogeneity $\leq 2$.

Theorem 5.1. Let $M$ be a simply connected Riemannian manifold on which a closed connected subgroup $G$ of the isometry group acts non-transitively and non-trivially. Let Gp, $p \in M$, be a principal orbit and assume that the isotropy group $K=G_{p}$ acts with cohomogeneity two on $M$. Then the $G$-action has no exceptional orbits and at most two singular orbits, which are fixed points. If there is a singular orbit, then the principal orbits $G / K$ are spheres. Else the principal orbits are simply connected rank-one symmetric spaces, i.e. isometric to one of

$$
\mathbb{R}^{n}, \mathrm{~S}^{n}, \mathbb{C P}^{n}, \mathbb{H} \mathrm{P}^{n}, \mathbb{O P} \mathrm{P}^{2}, \mathbb{R} \mathrm{H}^{n}, \mathbb{C H}^{n}, \mathbb{H} \mathrm{H}^{n}, \mathbb{O H}^{2} .
$$

More specifically, see Table 4:

(i) If there are no singular orbits then $M$ is a warped product $\mathbb{R} \times_{f} G / K$.

(ii) If there is one singular orbit then $M$ is isometric to $\mathbb{R}^{n+1}$ endowed with a rotationally symmetric metric, i.e. a metric invariant under a linear cohomogeneity one action of $G$ on $\mathbb{R}^{n+1}$.

(iii) If there are two singular orbits then $M$ is isometric to $S^{n+1} \subset \mathbb{R}^{n+2}=\mathbb{R}^{n+1} \times \mathbb{R}$ carrying a metric invariant under a linear cohomogeneity one action of $G$ on the $\mathbb{R}^{n+1}$ factor.

\begin{tabular}{|c|c|c|c|c|c|}
\hline No. & Singular orbits & $M$ & $G / K$ & Section $\Sigma$ & Fundamental domain \\
\hline \hline (i) & none & $\mathbb{R} \times_{f} G / K$ & $(\underline{5.2})$ & $\mathbb{R}$ & $\mathbb{R}$ \\
\hline (ii) & one point & $\approx \mathbb{R}^{n+1}$ & $\mathrm{~S}^{n}$ & $\mathbb{R}$ & $\mathbb{R}_{0}^{+}$ \\
\hline (iii) & two points & $\approx \mathrm{S}^{n+1}$ & $\mathrm{~S}^{n}$ & $\mathrm{~S}^{1}$ & {$[0, L]$} \\
\hline
\end{tabular}

TABLE 4. Inhomogeneous manifolds with unit tangent bundle of cohomogeneity one

The metric on the warped product $\mathbb{R} \times{ }_{f} G / K$ is $d t^{2} \oplus f(t)^{2} g_{0}$ where $f$ is a smooth positive function on $\mathbb{R}$ and $g_{0}$ is a fixed $G$-invariant metric on $G / K$. The metric $g_{0}$ is uniquely determined up to a scaling factor since $G / K$ is isotropy irreducible. All these manifolds are (degenerate) warped products, i.e. admit an isometry $I \times{ }_{f} G / K \rightarrow M$, where $I=\mathbb{R}, \mathbb{R}_{0}^{+}$or some finite interval $[0, L], L>0$, and $f>0$ on the interior of $I$ and $f=0$ on $\partial I=\emptyset,\{0\}$ or $\{0, L\}$, respectively. 
Proof. We fix a point $p \in M$, such that $G p$ is a principal orbit, and denote by $K \subset G$ the isotropy subgroup at $p$. The isotropy representation of $K$ splits as

$$
T_{p} M=T_{p} G p \oplus N_{p} G p .
$$

From this, it follows that $G$ acts with cohomogeneity one on $M$ and also that $K$ acts with cohomogeneity one on $T_{p} G p$. Let $\gamma: \mathbb{R} \rightarrow M$ be a normal geodesic such that $\gamma(0)=p$ and $\dot{\gamma}(0) \perp T_{p} G p$. Let $\Sigma=\gamma(\mathbb{R})$.

We prove the Theorem in the following steps:

1. Each $G$-orbit intersects $\Sigma$ perpendicularly. To prove this, let $G q$ be a second $G$-orbit and choose a geodesic $\psi$ realizing the distance of $G p$ to $G q$, say $\psi(0)=g p$ for some $g \in G$. This geodesic meets both $G p$ and $G q$ perpendicularly. Thus $g^{-1} \psi \subset \Sigma$ and $\Sigma$ meets $G p$ and $G q$ perpendicularly.

2. There are no exceptional orbits. If there are singular orbits, then these are fixed points and the principal orbits are spheres. Else there are no singular orbits and the principal orbits are simply connected. Let $G_{q} \subset G$ be the isotropy group at $q \in \Sigma$. Hence $K \subseteq G_{q}$. Since $\mathfrak{g}=\mathfrak{k} \oplus T_{p} G p$ and $T_{p} G p$ is irreducible, the Lie algebra $\mathfrak{g}_{q}$ of $G_{q}$ is either $\mathfrak{g}$ or $\mathfrak{k}$.

a) If $\mathfrak{g}_{q}=\mathfrak{g}$ then $G_{q}=G$ and $q$ is a fixed point.

b) If $\mathfrak{g}_{q}=\mathfrak{k}$ then $K$ has finite index $\iota$ in $G_{q}$. Assume $G q$ is an exceptional orbit i.e. $\iota>1$. The map $G p=G / K \rightarrow G q=G / G_{q}, g p \mapsto g q$, is well defined and has fibre $G_{q} / K$ with cardinality $\# G_{q} / K=\iota$. Thus it is a covering with $\iota$ sheets. Since $G p$ is connected, $\pi_{1}(G p)$ is a subgroup of positive index $\iota$ in $\pi_{1}(G q)$. We may assume $p$ arbitrarily close to $q$ since the union of the principal orbits is dense. By the Slice Theorem we may identify $G p$ with a submanifold of the sphere normal bundle $\mathrm{S} \nu(G q)$ of $G q$. Since $G p$ is connected we have

$$
G p=G / K=\mathrm{S} \nu(G q) \rightarrow G q=G / G_{q}
$$

a two-fold covering. We now apply the Theorem of Seifert-van Kampen to the decomposition

$$
M=\overline{D \nu(G q)} \cup M \backslash D \nu(G q)
$$

where $D \nu(G q)$ is a $G$-invariant open tubular neighborhood of $G q$ from the Slice Theorem. The intersection is

$$
\overline{D \nu(G q)} \cap M \backslash D \nu(G q)=\mathrm{S} \nu(G q)=G p .
$$

Thus $\pi_{1}(M) \cong \pi_{1}(G q) * \pi_{1}(M \backslash D \nu(G q)) / \pi_{1}(G p)$ cannot be trivial because $\pi_{1}(G p) \rightarrow$ $\pi_{1}(G q) \cong \pi_{1}(\overline{D \nu(G q)})$ is not surjective. Thus there are no exceptional orbits and singular orbits are fixed points.

If there is a fixed point then the distance sphere of a fixed point is a principal orbit. If there is no fixed point then all orbits are principal. Thus $M$ is a $G / K$-bundle over a one-dimensional manifold which is either $\mathbb{R}$ or $\mathrm{S}^{1}$. If it were $\mathrm{S}^{1}$ or if the fibres $G / K$ were not simply connected then so would be $M$, by the homotopy sequence $0=\pi_{2}(G \backslash M) \rightarrow$ $\pi_{1}(G / K) \rightarrow \pi_{1}(M) \rightarrow \pi_{1}(G \backslash M) \rightarrow \pi_{0}(G / K)=0$ of the fibre bundle $G / K \rightarrow M \rightarrow G \backslash M$. 
3. $M$ is a (possibly degenerate) warped product. For $g \in G$ we have that $g p \in \Sigma$ implies $g \Sigma=\Sigma$, because $\Sigma$ is the image of a geodesic $\gamma_{v}$ emanating from $p=\gamma_{v}(0)$ with $\dot{\gamma}_{v}(0)=$ $v \in T_{p} G p^{\perp}=T_{p} \Sigma$. Hence $g v \in T_{q} G p^{\perp}=T_{q} \Sigma$ and therefore $g v= \pm \dot{\gamma}_{v}\left(t_{q}\right)$ if $q=\gamma_{v}\left(t_{q}\right)$. Let $W=\{g \in G \mid g \Sigma=\Sigma\}$.

If there are no fixed points then $M$ is a fibre bundle over $G \backslash M=W \backslash \Sigma$. Since $M$ is simply connected we have $W \backslash \Sigma=\Sigma=\mathbb{R}$ and the $G$-orbits intersect $\Sigma$ only once. We thus have a diffeomorphism

$$
\begin{aligned}
\Sigma \times G p & \rightarrow M, \\
(s, g p) & \mapsto g s .
\end{aligned}
$$

Since the $G$-invariant metric $g_{0}$ on $G / K$ is unique up to scaling we can find a positive function $f$ on $\Sigma$ such that (5.3) becomes an isometry when $\mathbb{R} \times G / K=\Sigma \times G p$ is endowed with the warped product $d t^{2} \oplus f(t)^{2} g_{0}$. Thus $M$ is of the first type in Table 4 .

If there is a fixed point $q$ there is $w \in W \backslash K$ with $w^{2} \in K$ and $W$ is generated by $w$ and $K$. If $\Sigma=\mathbb{R}$ there are no other fixed point and if $\Sigma=\mathrm{S}^{1}$ we have at most two fixed points. In both cases the principal orbits are spheres. Away from the fixed points $M$ is a warped product over the interior of a fundamental domain $\Sigma_{0} \subset \Sigma$ for the $W$-action on $\Sigma$. This can only be $\Sigma_{0}=\mathbb{R}^{+}$or $\Sigma_{0}=(0, L)$ for some $L>0$, which produces the second and the third type in Table 4 , respectively. One has to replace $\mathbb{R}=\Sigma$ in (5.3) by the fundamental domain $\Sigma_{0}=\mathbb{R}^{+}$or $\Sigma_{0}=(0, L)$.

\section{REFERENCES}

[1] J. Berndt, F. Tricerri, L. Vanhecke: Generalized Heisenberg groups and Damek-Ricci harmonic spaces, LNM 1598, Springer, 1994

[2] I. Bergmann: Reducible polar representations. manuscripta math. 104 3, 309-324, 2001

[3] A. L. Besse: Einstein manifolds. Springer, 1987

[4] E.B. Dynkin: The maximal subgroups of the classical groups (Russian). Trudy Mosk. Mat. Obshch. 1, 39-166, (1952); English translation: Amer. Math. Soc. Transl. Ser. 2, 6, 245-378 (1957)

[5] S. Helgason: Differential geometry, Lie groups and symmetric spaces. Academic Press, 1978

[6] N. Jacobson: Lie algebras. Courier Dover Publications, 1979

[7] A. Kollross, F. Podestà: Homogeneous spaces with polar isotropy. manuscripta math. 110 4, 487-503, 2003

[8] A. Kollross, E. Samiou: Homogeneous spaces with sections. manuscripta math. 116 2, 115-123, 2005.

[9] C. Riehm: The automorphism group of a composition of quadratic forms, Trans. Amer. Math. Soc., 269 2, 403-414, 1982

[10] E. Straume: Compact connected Lie transformation groups on spheres with low cohomogeneity. I, Mem. Amer. Math. Soc., Vol. 119, No. 569, 1996. II, Mem. Amer. Math. Soc., Vol. 125, No. 595, 1997

[11] J. A. Wolf: The geometry and structure of isotropy irreducible homogeneous spaces. Acta Math. 120, 59-148, 1968; correction: Acta Math. 152, 141-142, 1984

Institut für Mathematik, Universität Augsburg, 86135 Augsburg, Germany

E-mail address: kollross@math.uni-augsburg.de

University of Cyprus, Department of Mathematics and Statistics, P.O. Box 20537, 1678 Nicosia, Cyprus

E-mail address: samiou@ucy.ac.cy 